\title{
Urological emergency activities during COVID-19 pandemic: Our experience
}

\author{
Elisa Cicerello, Mario S Mangano, Giandavide Cova, Alessio Zordani \\ Urology Unit, Department of Surgery, Ca' Foncello Hospital, Treviso, Italy.
}

\begin{abstract}
Summary Background: The Coronavirus Disease (COVID-19) is causing a significant health emergency which is overturning dramatically routine activities in hospitals. The outbreak is generating the need to provide assistance to infected patients and in parallel to treat all nondeferrable oncological and urgent benign diseases.

A panel of Italian urologists agreed on possible strategies for the reorganization of urological routine practices and on a set of recommendations that should facilitate a further planning of both inpatient visits and surgical activities during the COVID19 pandemic. According to this only urgent benign and nondeferrable oncological activities have been kept.

Materials and methods: We have considered urgent outpatient visits requested by Emergency Department (ED) or by General Practitioner (GP) and emergency surgical procedures performed in our Urology Unit from March $9^{\text {th }}$ to April $14^{\text {th }} 2020$, during COVID-19 pandemic. These figures have been compared to those observed last year from March 9th to April $14^{\text {th }}$ 2019.

Results: Our data show that urgent care visits decreased during COCID-19 pandemic (from 293 to 179). Urgent care visits of patients who accessed directly to the ED decreased (from 219 to 107) whereas the number of urgent care visits referred by GP remained unchanged (74 vs 72). Consequently, the rate of visits from $\mathrm{ED}$ decreased from $75 \%$ to $60 \%$ and the rate of visit requested by GP increased from $25 \%$ to $40 \%$ ( $p=0.001)$. Particularly, the rate of visits for renal colic, LUTS and other not precisely defined disorders from ED decreased and the corresponding rates of visits of patients referred by GPs increased significantly $(p=0.0001, p=0.0180$ and $p=0.0185$, respectively). The rate of visits for acute urinary retention, hematuria, sepsis, acute scrotum, cystitis, prostatitis and genito-urinary trauma from ED and GP remained unchanged. Finally, urgency endourology and surgical activities have been stable in relation to the same period last year.

Conclusions: Urological emergency activities during COVID19 pandemic are more appropriate since urgent outpatients' visits required by ED are decreased and emergency surgical and endourological procedures are stable.
\end{abstract}

KEY WORDS: Coronavirus disease 2019 (COVID-19); Pandemic; Emergency; Endourology; Surgery; Urology.

Submitted 26 May 2020; Accepted 12 June 2020

\section{INTRODUCTION}

Since December $31^{\text {st }} 2019$ Coronavirus Disease (COVID19) was notified by Chinese authorities and has been rapidly spreading worldwide until it reached the pandemic stage declared by WHO on March $11^{\text {th }}(1,2)$. After China, Italy was initially the country that was hit the hardest across Europe. The first case was documented on January $30^{\text {th }}$, but the emergency started only a few days later. Since March $8^{\text {th }} 2020$ following the Decreto del Presidente del Consiglio (DPCM), the whole country was on lockdown (3). By February $21^{\text {st }}$, the time when this paper was first drafted, 162.488 cases of COVID-19 have been diagnosed causing a total of 21.067 deaths, 28.011 critically ill patients, and 3.186 patients hospitalized in intensive Care Unit (ICU). In the Veneto region, whose first cluster was found on February $21^{\text {st }}$ in Vo' Euganeo (Padua), until today, 14.884 cases and 959 COVID-19 deaths have been reported (3).

Although this pandemic is causing an health emergency unprecedented in the Western countries, hospitals started facing the demand to dedicate all medical and logistical resources to the assistance of COVID-19 patients and to provide only treatments of urgent procedures or non-deferrable oncological interventions. This has inevitably caused a redistribution of all medical activities that are not directly involved in the management of COVID-19 patients. During the COVID-19 pandemic, urgent and non-deferrable activities have been kept as such, while non-urgent outpatient visits and surgical activities have been deferred until the end of pandemic (4). However, even those urgent operations have been limited, for instance, non-deferrable oncological operations in high anesthetic risk (ASA 3-4) patients became difficult to plan due to a lack of beds in intensive care unit (ICU). Thus, surgical treatments are reserved mainly to low anesthetic risk patients (ASA 1-2) for surgical procedures around renal (TCC), urinary tract urothelial cancer (UTUC), bladder and prostate cancer for high risk patients not eligible for radiation, while procedures for any other neoplasms have been postponed. When possible, alternative treatments for prostate cancer were proposed $(5,6)$.

This paper will report the experience of an Urology Unit (Azienda Sanitaria 2 - Marca Trevigiana) in Treviso, in the Veneto region, about urgent inpatient and outpatient activities during COVID-19 pandemic.

\section{MATERIALS AND METHODS}

For the aim of this observational study, we have considered all outpatients admitted to our Unit for urgent visit 
required by Emergency Department (ED) or by General Practitioner (GP) from March $9^{\text {th }}$ to April 14th 2020 , which include: renal colic, acute urinary retention, hematuria, urosepsis, genito-urinary trauma, acute scrotum, cystitis, prostatitis, urosepsis, low urinary tract symptoms (LUTS) and other not precisely defined urinary disorders. Emergency endourological procedures for obstruction of upper urinary tract, urosepsis or renal colic refractory to medicaments and surgical operations for acute scrotum or genito-urinary trauma have been analyzed. These figures have been compared to those visited between March $9^{\text {th }}$ and April 14 ${ }^{\text {th }} 2019$, a year before the COVID-19 outbreak. Statistical analysis was carried out by chi-square test as well as Student's test.

\section{Results}

These data show that urgent care visits requested by ED reduced during COVID-19 pandemic, if compared to those registered in the same time span last year (219 vs 107), while those addressed by GP were stable (74 vs 72). Consequently, the rate of visits from ED decreased from $75 \%$ to $60 \%$ and the rate of visit requested by GP increased from $25 \%$ to $40 \%$ ( $p=0.001$ ) (Table 1).

A reduction of urgent care visits of patients who accessed the ED for renal colic (-36), acute urinary retention (-8), hematuria (-12), cystitis (-10) and other not definitely disorders (-11) has been observed in the COVID period in comparison to the same period of the last year, while other urgent care visits for urosepsis $(=0)$, prostatitis $(+4)$, genitourinary trauma $(-2)$ and acute scrotum $(-2)$ remained stable.

Urgent care visits referred by GPs, instead, showed no difference for acute urinary retention $(+1)$, hematuria

\section{Table 1.}

Emergency outpatient visits required by Emergency Department (ED) and General Practitioners (GP) before and during COVID-19 pandemic.

\begin{tabular}{|l|ccc|ccc|c|c|}
\hline & \multicolumn{3}{|c|}{ Before COVID-19 } & \multicolumn{3}{c|}{ During COVID-19 } & Delta & Sig ED vs GP \\
\hline & ED & GP & Tot & ED & GP & Tot & & P \\
\hline Urgent inpatient visit & $219(75 \%)$ & $74(25 \%)$ & 293 & $107(60 \%)$ & $72(40 \%)$ & 179 & -114 & 0.0010 \\
\hline Renal colic & $56(85 \%)$ & $10(15 \%)$ & 66 & $20(54 \%)$ & $17(46 \%)$ & 37 & -29 & $<0.0001$ \\
\hline Acute urinary retention & $32(78 \%)$ & $9(22 \%)$ & 41 & $24(71 \%)$ & $10(29 \%)$ & 34 & -7 & NS \\
\hline Hematuria & $32(67 \%)$ & $16(33 \%)$ & 48 & $20(61 \%)$ & $13(39 \%)$ & 33 & -15 & NS \\
\hline Urosepsis & $12(60 \%)$ & $8(40 \%)$ & 20 & $12(55 \%)$ & $10(45 \%)$ & 22 & +2 & NS \\
\hline Acute scrotum & $11(65 \%)$ & $6(35 \%)$ & 17 & $9(64 \%)$ & $5(36 \%)$ & 14 & -3 & NS \\
\hline Cystitis & $11(55 \%)$ & $9(45 \%)$ & 20 & $1(50 \%)$ & $1(50 \%)$ & 2 & -18 & NS \\
\hline Prostatitis & $6(67 \%)$ & $3(33 \%)$ & 9 & $10(83 \%)$ & $2(17 \%)$ & 12 & +3 & NS \\
\hline Genitourinary trauma & $4(50 \%)$ & $4(50 \%)$ & 8 & $2(40 \%)$ & $3(60 \%)$ & 5 & -3 & NS \\
\hline LUTS & $38(76 \%)$ & $12(24 \%)$ & 50 & $3(33 \%)$ & $6(67 \%)$ & 9 & -41 & 0.0180 \\
\hline Other disorders & $17(94 \%)$ & $1(6 \%)$ & 18 & $6(55 \%)$ & $5(45 \%)$ & 11 & -7 & 0.0185 \\
\hline
\end{tabular}

\section{Table 2.}

Emergency endourological and surgical procedures performed before and during COVID-19 pandemic.

\begin{tabular}{|l|c|c|c|c|c|c|}
\hline & $\begin{array}{c}\text { Nephrostomy } \\
\text { tube }\end{array}$ & $\begin{array}{c}\text { Catheter } \\
\text { mono J }\end{array}$ & $\begin{array}{c}\text { Catheter } \\
\text { double J }\end{array}$ & $\begin{array}{c}\text { Testicular } \\
\text { torsion }\end{array}$ & $\begin{array}{c}\text { Genito-urinary } \\
\text { trauma }\end{array}$ & $\begin{array}{c}\text { Abscess } \\
\text { drainage }\end{array}$ \\
\hline Before COVID-19 & 3 & 3 & 6 & 1 & 1 & 1 \\
\hline During COVID-19 & 4 & 4 & 2 & 2 & 1 & 0 \\
\hline
\end{tabular}

$(-3)$, urosepsis $(+2)$, acute scrotum $(-1)$, prostatitis $(-1)$, genito-urinary trauma $(-1)$ between the pandemic period and the previous year.

An increase of visits for renal colic $(+7)$ and a decrease of visits for LUTS (-6) and cystitis (-8) have been registered. The rate of visits for renal colic, LUTS and other not precisely defined disorders from ED decreased and the corresponding rates of visits of patients referred by GPs increased significantly $(\mathrm{p}=0.0001, \mathrm{p}=0.0180$ and $\mathrm{p}=$ 0.0185 , respectively). The rate of visits for acute urinary retention, hematuria, sepsis, acute scrotum, cystitis, prostatitis and genito-urinary trauma from ED and GP remained unchanged.

Ultimately, there was no difference between the two periods for urgent endourological and surgical procedures (Table 2).

\section{Discussion}

COVID-19 pandemic has revolutionized clinical activities in several Urological Units. The unfortunate scenario of this pandemic required to address all health medical resources to COVID-19 patients. Hence, several Urological Units have been closing and urologists have been asked to support new COVID-19 wards that opened across the country.

According to urological guidelines $(5,6)$, in our Unit no urgent activities have been drastically cancelled. With regard to undeferrable oncological activities, the need of anesthesiologists, mechanic ventilator and intensive care beds for COVID-19 patients, has also delayed oncological operations in high risk patients (ASA 3-4). In fact, major surgeries such as radical cystectomy for bladder neoplasm, required in most cases post-operative surveillance in Intensive Care Unit (ICU), something that during the COVID-19 pandemic is not available.

This allowed us to treat low risk anesthetic patients (ASA 1-2) and perform only partial $(\mathrm{PN})$ or radical nephrectomy $(\mathrm{RN})$, radical nephroureterectomy (RNU), radical cystectomy (RC) or transurethral resection (TURB) for non-muscle invasive high risk bladder cancer and radical prostatectomy (RP) for high risk patients not eligible for radiation. Alternative therapies for other patients with prostate cancer have been proposed (radiotherapy or androgenic deprivation). All robotic and laparoscopic procedures have been cancelled according to COVID-19 recommendations to avoid virus widespread and to reduce or optimize operating time (7). All inpatient and outpatient procedures for benign diseases have been deferred until the end of the COVID-19 emergency, and only urgent activities have been preserved.

In our experience, the dramatic decrease of urgent outpatients' visits addressed by ED during COVID-19 pandemic could suggest that those are often non necessary, while those recommended by GP seemed to be 
more appropriate since they required emergency procedures. For instance, urgent visits for hematuria were required by GP only in cases of gross hematuria with clot retention and anemia, such as in the case of urological neoplasms or radiotherapy pelvis. Hospital admission with a quick correction of anemia and vesical catheter placement, and local irrigation of hemostatic solution are necessary and radiological or urological procedures must be performed in cases those local treatments do not work on the patient. Urgent visit for hematuria required by ED decreased, probably because most of the cases were single and asymptomatic episodes: in such cases people's fear and COVID-19 lock down reduced ED admission. This suggests that single and asymptomatic hematuria could be evaluated by sonogram or computerized tomography (CT) at home and urological follow-up should be postponed. Thus, unmodified urgent visit for prostatitis by ED have been addressed only for symptomatic prostatitis (strong urgency and frequency, urinary retention, prostate abscess and fever) that need urgent hospitalization: cystostomy, catheter placement and appropriate injecting antibiotic therapy are necessary to avoid urosepsis. Urgent visits for prostatitis addressed by GP have been stable probably because GP has continued to treat common and uncomplicated prostatitis at home. Besides, urgent inpatient visit for acute scrotum (testicular torsion or scrotal abscess), genito-urinary trauma and urosespsis, required both by GP and ED are unmodified during COVID-19 pandemic because they should require urgent endourological or surgical treatments. Overall, the decrease of urgent visits for acute urinary retention required by ED has been difficult to understand.

A hypothesis could be that strong dysuria is often confused with urinary retention and during COVID-19 lock down, when dysuria was not considered urgent problem, patients did not show up to ED at all. On the contrary, urgent outpatient visit addressed by GP for urinary retention before and during pandemic were stable because considered more urgent, which could explain the difference between the two group. Furthermore, the decrease of outpatient urgent visit for cystitis required both by GP and ED could suggest that these can be successfully treated with adequate antibiotic therapy and other preventive measures avoiding urological visit. Only in case of relapses or fever, non urgent urological evaluation could be performed and radiological follow-up required. The decrease of LUTS visits, both commissioned by ED and GP could suggest that this is usually a "misnomer": they are not specific urinary symptoms and cannot imply urgent urological diseases. Finally, urgent visit of other non-definite disorders might have been due to variations in code status limitations.

The reduction of urgent inpatient visits for renal colic sent by ED suggests that these are not necessary and renal colic could be confused with flank pain of other origin; GP, instead, sent patients mainly showing symptomatic urolithiasis (obstruction of the upper urinary tract, urosepsis or acute pain refractory to medical therapy): almost everyone was admitted to hospital where urgent endourological procedures were required. A percutaneous nephrostomy in local anesthesia was per- formed in case of severe obstruction of the upper urinary tract and fever. Ureteral catheter Mono J was used with severe sepsis or in anticoagulant therapy patients, while catheter Double J was placed in most of the cases that showed renal colic refractory to medical treatment. When possible, uncomplicated stones located in pelvic ureter were removed, while in all other cases, patients were discharged with ureteral stent Double J and the treatment was deferred until the end of COVID-19 pandemic.

Surgical procedures were performed for acute scrotum (testicular torsion, trauma or abscess) or genito-urinary trauma that cannot benefit from conservative treatment or radiological embolization. All these procedures performed during COVID-19 pandemic were not different from these required in emergency during the same period last year because urgent urological problems were unaltered. Endourological and surgical procedures were performed by expert urologists to minimize time and complications (8). Every patient even if asymptomatic underwent COVID-19 screening before emergency procedure. Enhanced Recovery After Surgery protocols has been adopted after each treatment and during hospital stay (9-11).

We could assert that emergency urological procedures during COVID-19 were stable, only urgent outpatient visits were registered and this is probably due to people's fear of COVID-19 as well as to the strict lockdown imposed by DPCM, which order to "STAY AT HOME". Additionally, General Pratictioners' activity has not diminished, on the contrary, it continues to take care of patients identifying those who really need hospitalization. This observation suggest that some patients could even be successfully treated at home by GP.

\section{Conclusions}

Even though Urologists are not involved in the first line during the COVID-19 pandemic, patients treated with urological emergencies are still present and consistent. Urgent urological procedures become necessary especially in episodes of complicated urolithiasis or testicular torsion or genitourinary trauma, in order to prevent obstructive renal failure and urosepsis and the loss of organs, respectively.

More specifically, the risk of underestimated patients with complicated urolithiasis seems to be low or absent since symptomatic renal colic have sent to urological unit by GP.

In conclusion, COVID-19 pandemic has suddenly revolutionized Urological activities, but it can also teach how to use ED properly after this pandemic.

\section{References}

1. Soharabi C, et al. World Health Organization declares global emergency: a review of the 2019 novel coronavirus (COVID-19). Int J Surg. 76:71-76.

2. WHO. Novel Coronavirus (COVID-199 situation. WHO https./experience argis.com/experience/685d0ace52164f8a5beeeee $1 b 9125 \mathrm{~cd}$. 
3. Decreto del Presidente del Consiglio dei Ministri 8 Marzo 2020: Ulteriori disposizioni del decreto-legge 23 febbraio 2020. n. 6, recante misure urgenti in materia di contenimento e gestione dell'emergenza epidemiologic da COVID-19. (2001522)(GUSerie Generale $n .59$ del 08-03-2020).

4. Italian Ministry of health. Nuovo coronavirus.www. salute.gov.it/ nuovo coronavirus.

5. Ficarra V, Novara g, Abrate A, et al. Urology practice during COVID-19 pandemic. Minerva Urol Nephrol. 2020; 72:369-375.

6. European Association of Urology Guidelines. Available at https:/uroweb.org/guidelines.

7. Zhen MH, Boni L, Fingerhut A. Minimally invasive procedures on the novel coronavirus outbreak: lessons learned in China and Italy. Ann Surg. 2020; 272:e5-e6.
8. Porpiglia F, Checcucci E, Amparore D, et al. Slowdown of urology resident's learning curve during COVID-19 emergency. BJU Int. 2020;,125:E15-E17.

9. Enhnanced Recovery After Surgery Society Guideline. Available at https: /erassociety.org/guidelines/list -of-guidelines/

10. Leonardi R, Bellinzoni P, Broglia L, et al. Hospital care in Departments defined as COVID-free: A proposal for a safe hospitalization protecting healthcare professionals and patients not affected by COVID-19. Arch Ital Urol Androl. 2020; 92:67-72.

11. Tafuri A, Minervini A, Celia A, et al. Comment on: Hospital care in Departments defined as COVID-free: A proposal for a safe hospitalization protecting healthcare professionals and patients not affected by COVID-19. Arch Ital Urol Androl. 2020; 92:80-81.

\section{Correspondence}

Elisa Cicerello, MD (Corresponding Author)

elisa.cicerello@tin.it

Mario S, Mangano, MD

Giandavide Cova, MD

Alessio Zordani, MD

Urology Unit, Department of Surgery, Ca' Foncello Hospital, Treviso, Italy. 